\title{
El problema de Smith y la relación entre moral y economía
}

\author{
José Atilano Pena López y José Manuel SÁnchez Santos \\ Departamento de Economía Aplicada I, Universidad de A Coruña
}

\begin{abstract}
RESUMEN. En el presente trabajo se replantea el denominado «A. Smith's problem» como base para una reconsideración de la interrelación entre Ética y Economía. A partir de la revisión crítica de los intentos de integrar la obra de Smith, se propone una alternativa de solución construida sobre los conceptos de simpatía y espectador imparcial. En particular, se pone de manifiesto que la moral entendida como extensión de relaciones de simpatía constituye una precondición para la existencia de cualquier forma de mercado. El análisis llevado a cabo permite aclarar cómo la moral constituye una base del sistema económico y cómo éste, a su vez, condiciona la propia moral.
\end{abstract}

Palabras clave: Ética y Economía, problema de Smith, simpatía.
ABstract. In the present paper we tried to put the denominated «A. Smith's problem» as a base to reconsider the interrelation between Ethics and Economics. Firstly we make a critical revision on the attempts to integrate the Smith's works on morals and economy, secondly we propose an alternative solution constructed on the smithian concepts of sympathy and impartial spectator. In our approach, moral is built as an extension of sympathy relations and constitutes a precondition for the market existence. The analysis carried out allows to clarify the way morals constitutes a base of the economic system and, at the same time, how economy conditions morals.

Key words: Ethics and Economy, Smith's problem, sympathy.

\section{Introducción}

La Economía neoclásica tiende a prescindir del hecho de que las interacciones económicas precisan algún modo de comprensión entre individuos o de una prosocialidad elemental ${ }^{1}$. En particular, el denominado paradigma de homo oeconomicus no ha prestado demasiada atención a la capacidad empática del hombre y a la existencia de vínculos de simpatía, aunque constantemente reconoce que los participantes en el mercado pertenecen a grupos que comparten ideas, sentimientos, valoraciones, etc. La denominada revolución marginalista y la consiguiente generalización del paradigma fisicalista en

${ }^{1}$ Existe una línea de investigación que trata de introducir en el análisis económico esta dimensión relacional a través del estudio de las funciones de utilidad interdependientes y la Teoría de Juegos. Al respecto puede verse Pena y Sánchez (2006, pp. 55-73). 
Economía, supuso a la postre una tendencia a forzar su escisión de las restantes ciencias sociales. Tanto es así que cualquier interpretación en clave mainstream consideraría fuera de lugar una proposición que afirmase que la Economía es una ciencia moral.

De hecho, la afirmación de que la Ciencia Económica tiene su punto de arranque en la obra de Smith y la caracterización de éste como una ruptura con la filosofía moral es uno de los tópicos más generalizados en la historiografía oficial del pensamiento económico. No obstante, los característicos binomios positivo-normativo, eficiencia-equidad, que surgen en el proceso de divorcio entre la Economía y la Ética, carecen de sentido en la obra de Smith (Young, 1997, pp. 5-11). El funcionamiento de la economía sólo es posible dentro de un marco motivacional, cultural e institucional más amplio, es decir, la economía es un subsistema del sistema cultural. De ahí que, frente al intento de separar la filosofía moral o la ética de la Economía, se impone la necesidad de un estudio científico que contemple el análisis económico de la moral y el análisis moral de la economía. El punto de referencia para desarrollar el mencionado estudio lo puede constituir el denominado «problema de Smith», esto es, la aparente contradicción entre sus dos obras centrales: La Riqueza de las Naciones ${ }^{2}$ y La Teoría de los Sentimientos Morales ${ }^{3}$, contradicción que ha alimentado un debate recurrente tanto en el ámbito de la Economía como en la historia del pensamiento económico (Sen, 1986, p. 32).

En el origen de la controversia está la creencia de que la $\mathrm{RN}$ ofrece una perspectiva incompleta en la que el ser humano parece guiarse exclusivamente por el interés propio, mientras que en la TSM proporciona una visión notablemente más compleja de la estructura motivacional del comportamiento humano, en la que valores como la justicia, la generosidad o el espíritu cívico desempeñan un papel esencial. En realidad, la obra smithiana en su conjunto proporciona una visión equilibrada de la relación entre Ética y Economía, que a la postre resulta de interés para una reconsideración de la articulación entre ambas dimensiones del actuar humano. La TSM y la RN se plantean como un intento de dar una respuesta sistemática a dos de los grandes problemas intelectuales de la ilustración inglesa. Por un lado, el debate altruismo-egoísmo existente entre los filósofos y teólogos del momento y, por otro, la preocupación de empresarios y administradores públicos por el incipiente crecimiento económico a escala nacional (Danner, 1976, p. 307).

Ambas obras estarían escritas desde dos puntos de vista considerados por Smith complementarios. La TSM se centra en los aspectos psicológicos de la

2 Smith, A. (1776) Investigación sobre la naturaleza y causas de la riqueza de las nacio-

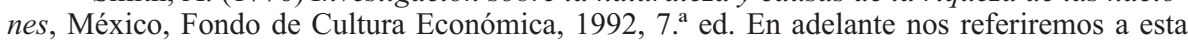
obra con la abreviatura RN.

3 Smith (1759) La teoría de los sentimientos morales, Madrid, Alianza, 1997, en adelante se aludirá a la misma con las siglas TSM. 
vida social y, por tanto, en sentimientos, pensamientos y tendencias naturales de socialidad. La RN, por su parte, aborda los aspectos económicos de la vida social, donde lógicamente las dimensiones psicológica o moral están aparentemente ausentes (Brown, 1994, p. 26). En esta línea, resulta pertinente un replanteamiento del «problema de Smith» que nos acerque al auténtico significado de la obra de este autor y que sirva de base para abordar la tensión existente entre el orden moral siempre tendente hacia la benevolencia y la «aparente» amoralidad de los mercados.

Los intentos de integración de la obra smithiana pueden clasificarse estableciendo una triple división. En primer lugar, nos encontramos con la interpretación que postula una moral construida sobre las relaciones de simpatía y que delimita el marco o las reglas básicas de juego a las que debería someterse del interés particular y, por tanto, de carácter exógeno a las propias relaciones económicas. En segundo lugar, cabe identificar una línea de análisis que considera la simpatía como un vínculo que delimita un entorno para cada individuo o una esfera en la cual no existen relaciones basadas en el interés particular sino de interdependencia o comunidad. Finalmente, está la postura que caracteriza la simpatía como un vínculo común presente con diversos grados en cualquier modo de relación y con efectos en la reducción de los costes de transacción. Los dos primeros enfoques, en última instancia, desplazan a ámbitos más restringidos el problema de Smith, dado que uno entiende la moral como un mero sistema corrector externo a las mismas relaciones económicas y el otro limita la moral y el altruismo a círculos ajenos al mercado. Por su parte, de acuerdo con la tercera de las perspectivas, tanto el interés particular como los vínculos de simpatía operan simultáneamente, predominando uno u otros en función de la distancia social entre los sujetos.

El presente trabajo se estructura como sigue. En el segundo apartado se exponen las bases del problema de Smith y se profundiza en el análisis de los conceptos de simpatía y espectador imparcial dada la importancia que ambos revisten a la hora de reconstruir la perspectiva de Smith sobre la articulación la articulación entre Ética y Economía. En el tercer apartado se revisan críticamente los diversos intentos de integrar el conjunto de la obra smithiana para acabar proponiendo la alternativa de respuesta al problema y, por extensión, a la dicotomía Ética-Economía, que consideramos más consistente. Finalmente, se resumen las principales conclusiones.

\section{El problema de A. Smith}

Como punto de partida para abordar el problema de Smith cabe mencionar que este autor, como antes lo había hecho Hume, recupera una tradición de estudio que busca un primer principio de la moral de la que participan pensadores de muy diverso signo, desde Spinoza a Mandeville. Cronológicamente, 
la primera de las obras de Smith (TSM) ${ }^{4}$, presta su máxima atención a la formación de los juicios morales de nuestras acciones y de las acciones de otros, siendo las nociones de prudencia y simpatía el núcleo de la argumentación. Por su parte, en la RN se preocupa por el estudio de los factores conducentes al desarrollo económico y apunta al interés particular como motor del mismo.

Así, en el comienzo de la (TSM) puede leerse

«Por más egoísta que se pueda suponer al hombre, existen evidentemente en su naturaleza algunos principios que le hacen interesarse por la suerte de otros, y hacen que la felicidad de estos resulte necesaria, aunque no derive de ella más que el placer de contemplarla» (TSM, I,1).

En tanto que en el fragmento más citado de la RN se afirma:

«No de la benevolencia del carnicero, del cervecero o del panadero de quien esperamos nuestra cena (...) sino de su interés particular...» (RN, I, 2).

De una lectura descontextualizada de los fragmentos más citados de ambas obras parece desprenderse la existencia de una contradicción básica entre su obra inicial de carácter ético y su posterior obra económica. Ahora bien, lo sorprendente es que esa contradicción no fue objeto de debate en la época en la que Smith desarrolla su labor educativa, sino que aparece un siglo más tarde.

La primera alusión histórica al «problema» surge entre pensadores alemanes de orientación socialista fuertemente críticos con el librecambismo dominante. Estos autores, entre los que destaca Skarzynski, afirman que en la obra de Smith existe una doble interpretación de la naturaleza humana que resulta contradictoria (Peters-Fransen, 2001). La benevolencia o el altruismo ${ }^{5}$ defendidas en la TSM serían incompatibles con la defensa del egoísmo como fundamento del sistema económico que se hace en la RN. Oncken ${ }^{6}$, Brentano y Knies, atribuyeron esta contradicción al giro (umschwungstheorie) que habría supuesto para Smith el contacto con el pensamiento liberal francés de los filósofos materialistas (Helvetius, Holbach...) y las ideas de los fisiócratas que tendrían su refrendo en la RN, frente a la influencia que Hume y Hutcheson habían tenido en su obra moral (Nieli, 1986; Teichgraeber, 1986).

${ }^{4}$ Esta obra estableció su reputación como filósofo en toda Europa y su impacto de su obra moral en el pensamiento de la época no debe ser subestimado. De hecho tuvo cinco ediciones en vida y fue inmediatamente traducida al francés (tres veces en el XVIII) y al alemán (dos veces en el mismo siglo) y fue leída y discutida por todos los grandes filósofos del XVIII y XIX, desde Hume hasta Kant. Sobre este tema puede verse el estudio de Reeder (1998, pp. 9-39).

${ }^{5}$ El término altruismo está ausente de toda la obra de Smith. No podía ser de otro modo, ya que se trata de una invención de A. Comte, un siglo más tarde. La expresión al uso en el mundo intelectual smithiano era heredera de la tradición escolástica y no exactamente equivalente: «Amor de benevolencia».

${ }^{6}$ Entre las obras de este grupo de autores merece la pena destacar la postura de Oncken (1897). Este autor fue el que acuñó la expresión «Das A. Smith problem». 
Esta explicación mantiene su vigencia hasta bien entrado el siglo $\mathrm{Xx}$, en el que autores de la talla de J. Viner siguen manejando esta hipótesis como un lugar común (Viner, 1927), de forma que el origen de la Economía como ciencia tendría como punto de partida un «desengaño» de Smith con respecto a ciertas veleidades morales. En esta línea, cabe traer a colación la interpretación reduccionista de Becker (1981). Este autor señala que la introducción que Smith realiza a sus dos obras sugiere que en tanto el egoísmo es común en las transacciones de mercado, el altruismo lo es dentro de las familias, pero no explica por qué las mismas personas son altruistas en las familias y egoístas en el mercado. Desde una interpretación descontextualizada, Becker explica esta dualidad por motivos puramente de eficiencia. La misma idea es retomada por Kolm (1983) al afirmar que la Economía como ciencia se asienta en la denominada «falacia Smith-Mandeville», según la cual una economía basada en relaciones de carácter altruista sería menos eficiente que una basada en agentes egoístas. Este planteamiento lleva a algunos autores a situar en Smith uno de los hitos claves de destrucción de la moral (Lux, 1990). Una lectura parcial de la obra de Smith también está presente en la visión más generalizada dentro de la Escuela Austriaca, para la que la obra smithiana es un cúmulo de errores. Éste es el caso de los comentarios de Rothbard (2003, pp. 519ss.) sobre el «problema de A. Smith» donde no identifica correctamente su auténtica raíz y prescinde por completo de la dimensión ética y social de la obra smithiana ${ }^{7}$. En suma, tras la percepción de aquellos autores que reconocen la existencia del problema están los prejuicios de lectura ${ }^{8}$, presuponiendo que la moderna ficción del homo oeconomicus es el trasfondo propio de la RN (Alvey, 2003).

No obstante, las pruebas documentales vienen a poner en cuestión la hipótesis del giro de pensamiento. Por una parte, del contenido de los apuntes de las clases de A. Smith contemporáneas a la TSM, se desprende que las ideas matrices de la RN estaban ya presentes en su obra moral (Cannan, 1896; Rae, 1895) ${ }^{9}$. De hecho, el propio Smith no observó la existencia de ninguna incongruencia, pues en la última página de la TSM, adelanta que continuará en su próxima obra la tarea del estudio de los principios generales

\footnotetext{
7 En cierta medida la crítica a la obra de Smith por parte de estos autores resulta sorprendente en tanto que su visión moral del hombre y del papel desempeñado por la moral es notablemente semejante al austríaco. Además, Hayek consideraba la perspectiva smithiana como precursora del concepto de Catalaxia y él mismo se adscribía dentro del individualismo smithiano (Hayek, 1984). Sobre este tema puede verse el trabajo de Matthews (2000).

${ }^{8}$ La idea de que el problema es el resultado de una incomprensión es compartida por un amplio conjunto de autores entre los que destacan entre otros Macfie (1967), Winch (1978), Brown (1994), Fitzgibbons (1995) y Young (1997).

9 El curso de Filosofía Moral que Smith enseñaba en la Universidad de Glasgow en 1750 tenía tres partes: teodicea, ética y justicia. La última se centraba ya por entonces en cómo incrementar la riqueza de la nación. Veáse al respecto Dimand y Dimand (1991).
} 
de la ley, el gobierno y la política. Asimismo, en el anuncio de la sexta edición de la obra señala que la RN suponía el cumplimiento de lo expresado en la última página de su obra moral. Finalmente, en una especie de ultílogo Smith explicaba su deseo, cumplido sólo parcialmente, de escribir una filosofía social completa que abarcase las tres grandes áreas: Ética, Jurisprudencia y la más reciente de la Economía Política.

\subsection{La simpatía y el espectador imparcial en el sistema smithiano}

Smith percibe una diferencia esencial de lenguaje entre la Ética y la Economía y, en particular, el lenguaje de la Economía, que precisa ajustarse a la explicación de reglas de actuación, no es apropiado para el análisis del mundo interior del hombre. Sin embargo, a diferencia de sus contemporáneos que adoptan una perspectiva puramente racionalista o que basan sus análisis en la ficción teórica del Estado de Naturaleza, Smith no considera al hombre aislado, sino al hombre de la vida de relación. Bajo esta visión, los seres humanos son por naturaleza sociales en el estricto sentido aristotélico y tienen como fundamento de actuación el deseo de reconocimiento, de ser apreciados, aceptados, aprobados, e incluso amados por otros seres humanos (Ingrao y Ranchetti, 2000, pp. 60-61). Los individuos, mediante la simpatía, se ven afectados por el bienestar ajeno, especialmente si existe algún modo de relación con el otro. No se trata meramente de un contagio, una comunicación o una transferencia emocional, sino que el sujeto se emplaza en el lugar del otro (TSM, I, 1) a través de un acto de imaginación:

«Cuando yo me conduelo con otra persona por la muerte de su hijo no estoy suponiendo cómo me sentiría yo sino cómo se sentiría una persona de sus circunstancias, considero cómo me sentiría si realmente fuese el otro sujeto» (TSM, I, 2, 4).

Este vínculo, por tanto, supone un cambio de posiciones más reflexivo y complejo con importantes implicaciones cognitivas, esto es, afecta al proceso de toma de decisiones.

La definición del concepto smithiano de simpatía no es simple, dado que el autor lo reviste constantemente de cierto intuicionismo moral y lo hace omniabarcador, al incluir bajo la misma denominación diversos grados de vínculo cualitativamente diferentes. La ambigüedad resultante hace necesario un recorrido exegético por la obra a fin de profundizar en las distintas acepciones del término.

El pensamiento moral del momento confrontaba una visión de la naturaleza humana esencialmente egoísta (Maquiavelo, Hobbes, Mandeville...) a una ética asentada en la exaltación de la benevolencia (Shaftesbury, Hutcheson...). Smith opta, en cambio, por una vía intermedia en la que se rompe con el solipsismo mandevilliano y la moral de virtudes heroicas de Shaftesbury, 
para asentar la moral en las tendencias naturales del hombre y explicar su prosocialidad ${ }^{10}$ (TSM, I,1.4).

El hombre no deriva satisfacción o placer exclusivamente de sí mismo, sino también de la implicación en las experiencias de sus congéneres. Si bien es indudable que dispone de la capacidad de percibir un placer individual biológico, también experimenta otras formas de placer vinculadas a la interacción social (TSM, I, 1 y I, 2). Esta afirmación, que parece contradecir la moral egoísta de Mandeville, no es equivalente a la moral de benevolencia. No se trata de «amor al prójimo», ya que la conducta humana implica necesariamente en primer lugar la atención a los propios intereses, y sólo en segundo término la atención al prójimo.

«Es indudable que por naturaleza cada persona debe primero y principalmente cuidar de sí misma, y como cada ser humano está más preparado para cuidar de sí mismo antes que de cualquier otro, es correcto que asi lo sea. Por tanto, cada individuo está más interesado en lo que le preocupa a él que a cualquier otro» (TSM, II,2,2).

De igual manera, el vínculo de unión a los otros depende de una relación de proximidad o familiaridad, una especie de delimitación de ámbito propio en el que es posible la interdependencia de individuos, con lo que Smith sostiene la existencia de un sistema completo de prelaciones personales.

«Después de sí mismo, los objetos naturales de sus afectos más cálidos son los miembros de su familia, los que viven normalmente en su casa (...) aquellas personas sobre cuya felicidad o infelicidad más influencia puede ejercer su conducta» (TSM, VI, 2, 1).

Esta tendencia natural conlleva también la inducción o contagio de opiniones y actitudes; es decir, constituye la base para el consenso y la cohesión social. Los individuos aproximan sentimientos, afectos y emociones hasta lograr alguna forma de acuerdo, con lo que se fortalece y uniformiza el grupo.

«Conocer las opiniones ajenas supone adoptarlas parcialmente. Aprobar las opiniones ajenas es advertir su acuerdo con las nuestras» (TSM, I, 1).

"Cuando la naturaleza formó al ser humano para la sociedad lo dotó con un deseo original de complacer a sus semejantes y una aversión original a ofenderlos. Le enseñó a sentir placer ante su condición favorable y dolor ante su consideración desfavorable, su aprobación halagadora y su desaprobación ofensiva» (TSM. III, V, 1).

Ahora bien, contrariamente a lo que sugerían quienes señalaban las inconsistencias en el sistema de pensamiento smithiano, la simpatía no es traducible por benevolencia, dado que esta última tiene rango de virtud y es

10 Entendemos por prosociales aquellos comportamientos tendentes a generar alguna forma de comunidad entre los individuos, esto es, que favorecen la construcción de relaciones sociales. 
supererogatoria, es decir, no exigible, en tanto que la simpatía es un principio psicosocial connatural al hombre sobre el que se construye la vida en comunidad.

Smith señala que la simpatía es un acto de «ponerse en lugar de» (TSM. I, 1) y «padecer con» (TSM, I, 3, 1). En virtud de dicho acto y mediante ciertas habilidades imaginativas condicionadas por la proximidad, cada hombre forma una idea de cómo se sienten otros, considerando cómo se sentiría él en tales circunstancias y al mismo tiempo se identifica con ellos. De esta caracterización de la simpatía se pueden extraer tres implicaciones. Primero, se evidencia la doble dimensión del hombre ya que la simpatía no puede ser considerada un principio egoísta (TMS, VII, 3). Segundo, pone de manifiesto la generación de un vínculo con las experiencias ajenas (TMS, I, 1). Finalmente, destaca que no se trata sólo de una transferencia emotiva sino que es cognitiva y base de la aprobación moral (TSM, I, 1, 3 y TSM, I, 1, 4).

En términos epistémicos la definición de simpatía que plantea Smith tiene carácter subracional y, por tanto, no identificable con el pensamiento analítico, sino con un componente intuitivo, dado que es experimentada instantáneamente al margen de cualquier proceso de razonamiento. De hecho, sostiene que es totalmente absurdo suponer que la percepción de bondad o maldad moral pueda ser derivada de la razón, al igual que niega que estos sentimientos puedan ser deducidos del amor propio (TSM, I, 1). Se trata consecuentemente de un emotivismo moral.

En cierta medida surge aquí, a partir de la noción de simpatía, una teoría sobre una tendencia natural al intercambio de sentimientos y reconocimiento (Fuertes, 2000, pp. 160ss.), donde la corrección en el actuar es fruto de un equilibrio en la confluencia de dos factores distintos:

«El del espectador por identificarse con los efectos de la persona afectada y el de ésta por atenuar sus emociones hasta el punto que pueda acompañarla el espectador» (TSM, I, 2).

Esta idea podría interpretarse como un proceso de oferta y demanda de reconocimiento mutuo en el que ambos individuos intercambian sentimientos «hasta un punto intermedio o límite donde radica la corrección» (TSM, II, 1). El acuerdo oferente-demandante es equivalente al acuerdo entre espectador y protagonista de la acción. Así, al igual que el equilibrio de mercado surge de la confrontación de intereses particulares en la búsqueda de la satisfacción de la demanda, la moral sigue un movimiento semejante en la búsqueda de la aprobación social. Consiguientemente, los individuos smithianos buscan un progreso material necesario, pero también la aprobación moral, y ambas búsquedas, en virtud de un principio de autoconservación ${ }^{11}$ son análogas en cuanto proceso social de harmonización (Fuertes, 2000, p. 173).

${ }^{11}$ La filosofía estoica, de la que Smith y el mismo kantismo son herederos, había planteado igualmente como primer principio de todo sistema moral la autoconservación o oikeosis. 
Así pues, la simpatía constituye una base de naturaleza psicológica, tanto de la construcción de la sociedad y la moral, como de la misma economía. Consecuentemente, mientras la ética se centra en la noción de simpatía entendida como la capacidad de juicio de la propia acción desde el punto de vista del otro, la segunda lo hará en el valor de cambio y del comercio que igualmente precisa la ayuda de los otros para ser efectivo. En cierta medida, podría afirmarse que el giro radical en la obra de Smith reside en que la moral se explica como una teoría de la interacción social y se sustenta sobre una definición de utilidad como interdependencia. Este interaccionismo acabará por favorecer la precoordinación de las decisiones individuales en virtud de la interdependencia social que genera la simpatía. En conjunto, Smith está planteando una geometría de las relaciones humanas como un sistema de equilibramiento mutuo, muy semejante a un sistema gravitacional newtoniano ${ }^{12}$, pero también muy semejante a la actividad comercial.

La simpatía es también la base para la formación de los juicios morales, ya que a través de ésta los hombres son capaces de superar su natural condición de autocentramiento y de verse como otros los ven. Sin embargo, como fuente de conocimiento de la situación de los otros, es un principio insuficiente para asentar un sistema moral. Así, junto a la simpatía aparece un constructo derivado, un imaginario creado en nuestra mente, al que Smith denomina «Espectador Imparcial» y que se erige en un juez de nuestra conducta. Se trataría de la mirada imparcial de una tercera persona (la conciencia) por la que el individuo se siente llamado a decidir lo que le hace auténticamente humano mediante la búsqueda de aprobación exterior (TSM, III, 2). De esta capacidad se deriva que el hombre es capaz de observar imparcialmente tanto sus propias acciones como las de los restantes individuos.

Puesto que el espectador juzgará tanto la conducta ajena como la propia, está planteando un constructo muy similar al Superego freudiano, una especie de interiorización de las normas sociales producto de un proceso de socialización. En la medida en es capaz de examinar un acto o situación desde la distancia, los juicios y valoraciones individuales no son independientes de medio sociocultural en el que se inserta el individuo (TSM, III, 1,6).

«Si fuera posible que una criatura humana pudiese desarrollarse en un paraje aislado, le sería tan imposible pensar en su propia personalidad, en la corrección de sus sentimientos y su conducta, en la belleza (...). Todo ello es producto de la interacción social» (TSM, III, 2).

Para Smith este espectador adopta la perspectiva del grupo social (una internalización de los valores sociales) en el que se integra el individuo y no se

12 Ha existido una notable discusión sobre el punto hasta el que Smith llegó a verse influenciado por el pensamiento newtoniano. Lo que no es objeto de duda, sin embargo, es el hecho de que Newton se había convertido en una especie de «divinidad tutelar» del pensamiento del momento (Wightman, 1975). 
limita a valorar una acción en función de la repercusión sobre su propia utilidad (Khalil, 2001, pp. 425-426). En último término, el espectador imparcial representa la posición de una imparcialidad bien informada que puede corregir las distorsiones de juicio en las que inevitablemente incurren los espectadores reales (TMS, III, 3). Precisamente, esta característica sitúa al espectador imparcial en la base de toda norma social.

En síntesis, los seres humanos se preguntan sobre la moral de los actos cuestionándose por simpatía sobre las repercusiones de los mismos y juzgan sus propias acciones y las de los demás planteándose cómo serían valoradas por un espectador imparcial, una abstracción del grupo en la propia mente del sujeto (Raphael, 1975, pp. 83-99) ${ }^{13}$.

Buena parte de la literatura sobre la teoría moral de Smith pasó por alto esta distinción. Por ejemplo, el hombre primario de Heilbroner (1982, pp. 427-439) está impulsado por el deseo de recibir reconocimiento y aprobación, lo que lo conduce a un proceso de socialización que hará que sea prudente o beneficente de acuerdo a las normas morales. También Skinner señala que Smith estaría fundamentándose en una tendencia natural de acomodación a la sociedad (Skinner, 1971). En una línea semejante y adoptando una perspectiva evolutiva, para Campbell la conciencia (espectador imparcial) tiene su origen en una maduración individual que capacita al sujeto para percibir su propio comportamiento a fin de anticipar las reacciones a éste y de interiorizar las normas morales del colectivo en el que se inserta (Raphael, 1975) ${ }^{14}$. Sin embargo, la propuesta de Smith se puede catalogar más bien como una orientación altercéntrica, es decir, el espectador imparcial se constituye como un sujeto exterior que tiene a su cargo la valoración de las actuaciones, cumpliendo las funciones que la moral tradicional ha atribuido a la conciencia. De este modo y en último término quien valora es la sociedad o el grupo de identidad del individuo. Este aspecto pone de relieve la penetración psicológica de la obra de Smith hasta tal punto que resulta difícil distinguir dónde comienza el filósofo moral y acaba el psicólogo social ${ }^{15}$.

13 Sobre este tema es especialmente interesante el artículo de Khalil (1990).

14 Aunque existen algunas similitudes obvias no puede hacerse una identificación simplista del espectador con el superego freudiano manejada por Raphael. Sin embargo, tanto este autor como Campbell consideran la autonomía del estadio ternario desde una perspectiva evolutiva al afirmar que la conciencia que surge inicialmente como adopción de la opinión pública, gana autonomía en la medida en que la persona madura.

15 Podemos encontrar infinidad de trabajos en psicología en los que se adopta la misma perspectiva desde la orientación psicoanalítica y el estudio del «super-yo». En éstos se insiste en que la visión altercéntrica o el nacimiento de la conciencia es un resultado de la evolución de la percepción egocéntrica propia de los estados infantiles. 


\section{Alternativas de disolución del «Problema de Smith»}

\subsection{La simpatía y la moral como marco o reglas de juego del interés particular}

Para un importante grupo de autores (Anspach, 1972; Wilson, 1975; West, 1976) entre los que destaca especialmente Campbell (1967), la simpatía de la TSM constituye la base sobre la que se asienta el sistema moral, político y judicial, imprescindible para el funcionamiento de una sociedad de individuos guiados por el interés particular. Dicho de otro modo, la simpatía es la base de un sistema moral que establece las reglas de juego a las que deberían someterse los intereses particulares. Por tanto, para esta línea interpretativa, al igual que Mandeville (1714) en La fábula de las Abejas defendía la necesidad de un sistema judicial apoyado en la moral que «envolviese» los vicios benéficos, Smith construiría el orden moral y sobre éste el legal, como el marco de control de la actividad económica.

El problema central tanto para Smith como para Mandeville residiría en cómo canalizar el interés particular hacia manifestaciones socialmente benéficas. Así, prudencia y justicia, las dos mayores restricciones presentes en la TSM lo están igualmente en la RN, en tanto que la virtud por excelencia, la benevolencia, está totalmente ausente, ya que ésta es una consecuencia más que una causa del crecimiento económico. De acuerdo con esta lectura, Smith no otorga primacía al interés particular o al egoísmo ni en la TSM ni en la RN. En realidad, en la TSM busca establecer el apropiado marco institucional en el que el interés particular puede expresarse sin dañar a los individuos.

Desde esta perspectiva, todo sistema moral debe tener su traducción normativa y una acción no puede ser calificada de socialmente benéfica si supone daño hacia otros individuos. Dichas acciones potencialmente nocivas deben ser evitadas mediante la formulación de normas morales colectivamente asumidas, para las que, dada la dificultad de lograr un acuerdo unánime, la mejor forma de promulgación es el consenso. Así, el establecimiento de normas a partir de las virtudes antes mencionadas de prudencia y justicia acaba materializándose por un lento proceso de consenso social de modo semejante a la creación de las normas lingüísticas y gramaticales.

De este modo, el papel del espectador imparcial, guiado por un mecanismo psicológico de simpatía, se reduce a favorecer un desarrollo moral del individuo que impida los desórdenes consecuencia del libre ejercicio del interés particular.

En último término esta interpretación se asienta en una defensa del sistema smithiano de libertad y justicia naturales, en donde

«Todo hombre, en la medida en que no viole las normas de justicia, sea perfectamente libre de perseguir su propio interés e introducir su industria y 
capital en la competencia con cualquier otro hombre u orden de hombres» (RN. 651).

Indudablemente, la moral smithiana se contrapone a los rígidos sistemas de restricciones mercantilistas; de hecho, los libros III y IV de la RN son una constante demanda de imparcialidad y libertad para el orden social (Campbell, 1967, pp. 571-577).

En una línea similar, Macfie (1967, p.75) subraya que la aparente contradicción de la obra smithiana surge de que la RN adopta una perspectiva más estrecha que la TSM, puesto que la primera analiza el subsistema económico mientras que la segunda se centra en el sistema social general. Así, el hombre económico de la RN, es el hombre prudente de la TSM actuando en el ámbito de mercado. En otras palabras, el problema de la limitación del análisis de las motivaciones conductuales en la RN es una consecuencia del estudio de las peculiaridades de un marco concreto. A su vez, Morrow (1927) justifica la presunta contradicción afirmando que los comportamientos motivados por el interés particular en el ámbito del mercado también se encuentran bajo las fronteras de justicia definidas en la TSM y la «vigilancia del espectador imparcial». Para este autor, el problema se deriva en último término del hecho de que la RN es una especie de evangelio de la defensa de los derechos individuales típica del pensamiento del XVIII.

Desde nuestro punto de vista, en su conjunto esta primera alternativa interpretativa del problema de Smith adolece de los prejuicios que supone el acercamiento a las TSM desde la RN. La obra smithiana es interpretada como esencialmente económica y confrontada con el mercantilismo $\mathrm{y}$, de acuerdo con dicha interpretación, la moral delimita ámbitos de actuación pero no penetra en las relaciones económicas. Además, prescinde del concepto clave que subyace a toda la TSM, la noción de simpatía, centrándose en remarcar la importancia de los sistemas normativos como trasfondo de las relaciones económicas. Por otra parte, no resuelve el problema, porque simplemente señala la importancia del control externo del interés particular, pero no integra el papel de la moral que sigue siendo un corrector externo de las relaciones económicas.

\subsection{Las esferas de simpatía en la obra de Smith}

Existe una segunda línea interpretativa según la cual los sentimientos morales establecen fronteras que delimitan el ámbito de actuación del interés particular, lo que podríamos denominar esferas de simpatía o intimidad (Hollander, 1973; Nieli, 1986). A esta interpretación subyace la distinción, típica de la tradición británica, entre esferas públicas y privadas. La simpatía y benevolencia se asocian a un marco más estrecho de familia y amigos, en tanto que el mercado constituye una sociedad de extraños en la que la simpatía smithiana es desplazada por las virtudes de prudencia y autocontrol (Teichgraeber, 1986). 
En una primera lectura, la interpretación del funcionamiento de la economía que realiza Smith parece particularmente estrecha, dado que las relaciones económicas son intercambios de bienes y servicios entre personas que carecen de lazos personales; es decir, observa el mercado como la interacción de mónadas aisladas. Sin embargo, de una lectura más profunda parece desprenderse que realmente percibe el mercado como la interrelación de grupos primarios, de forma que en la $\mathrm{RN}$ aborda exclusivamente las relaciones sociales entre miembros de comunidades diversas entre las que no existen vínculos personales. Esta afirmación es perfectamente verificable cuando alude a las metáforas de comportamiento prudente de los padres de familia y de las familias como unidades de decisión (RN IV, II, 2) o cuando se refiere a los actores económicos en la cita más común de esta obra (panaderos, cerveceros, etc.) como agentes dentro de las interacciones del mercado, pero que, a su vez, son miembros de grupos de no-mercado. Los abundantes ejemplos dispersos por la RN permiten comprobar como Smith considera implícitamente que la simpatía opera junto al interés particular favoreciendo tanto la armonía social en general como la económica en particular (Danner, 1976).

De acuerdo con esta segunda visión, las relaciones de mercado no dominan los círculos en los que existe una cierta proximidad personal, sino que en estas esferas de intimidad se manifiestan especialmente las relaciones de confianza y simpatía. A su vez, dentro de éstas se establece una prelación u «orden en que los individuos son encomendados por la naturaleza a nuestro cuidado y atención» (TSM I, 2, 2.1). En este pasaje, se alude claramente a una gradación en la simpatía con los individuos que nos rodean, en razón de la «distancia social» que nos separa. Consecuentemente, las relaciones de mercado se ven mediatizadas por la existencia de este tipo de entornos delimitados. Desde un punto de vista filosófico, Smith traduce a términos socioeconómicos la idea estoica de que un hombre tiene como primer deber natural su

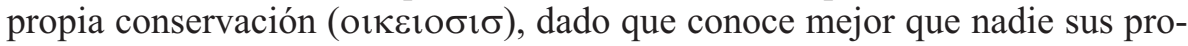
pias necesidades (TSM, VI, 2, 1.1). Fuera de sí mismo la preocupación y simpatía recae naturalmente sobre sus más allegados, aquellos con los que convive, tanto por el nivel de conocimiento como por su capacidad de actuar sobre ellos (TSM VI, 2, 1.2 y 1.5).

No obstante, a este respecto es importante recalcar que Smith no considera estos vínculos como identificables con lazos familiares, dado que los lazos sociales no se constituyen primordialmente por la consanguinidad, sino por contacto y proximidad y en especial, los individuos se sienten abocados por una relación de reciprocidad (quid pro quo) hacia aquellos cuya «simpatía» han percibido (TSM. VI, 2, 1.19). "Este afecto natural es más la consecuencia de la conexión moral que del supuesto nexo físico entre padres e hijos» (Ib.). Smith amplia progresivamente el diámetro de la esfera de simpatía y pasa a considerar «el orden en el cual los grupos nos son encomendados por naturaleza» (TSM, VI, 3). Por ejemplo, añade una especial consideración ha- 
cia los pobres y aquellos que dependen de nuestra compasión (TSM, VI, 2, 1.2). Finalmente, el último círculo al que los agentes pueden vincularse desde la perspectiva de Smith es el Estado. Cada persona tiene una especial relación con los miembros de su país, no sólo por la vinculación personal y cultural, sino también por el efecto de inclusión, puesto que la prosperidad de su país incide directamente sobre el bienestar de las esferas de intimidad a las que se siente referenciado (TSM,VI, 3, 2.1). Ahora bien, los vínculos con el Estado los limita al cumplimiento de la ley y el pago de impuestos. Esta concepción responde ya a la nueva visión utilitarista-liberal en la que el individuo no existe para el bien del Estado; sin embargo, incluso en este extremo el comportamiento de defensa del Estado sólo es explicable como una simpatía con un círculo de gran amplitud (TSM, IV, 2) ${ }^{16}$.

En definitiva, esta perspectiva adolece de cierto simplismo en lo que respecta al análisis textual de la TSM, en la medida que evita el problema diferenciando los ámbitos de aplicación de cada una de las obras, con lo que es equivalente a la extendida versión beckeriana de «altruismo en la familia, egoísmo en el mercado» ${ }^{17}$. Nuevamente, la moral es relegada al no-mercado y el problema de A. Smith sigue presente, trasladado ahora a la oposición entre las esferas de intimidad y el mercado. Por otro lado, el hecho de que la definición de esferas de simpatía no parecen suponer la existencia de límites definidos invita a plantear la extensión de las relaciones de simpatía frente al carácter restrictivo de esta segunda interpretación.

\subsection{La imbricación entre moral y economía en Smith o la simpatía como base del orden social}

En los intentos de integrar la obra de Smith, probablemente uno de los aspectos que reviste mayor complejidad es la interpretación de la noción de

16 Pese a todo, Smith no da el siguiente paso y considera inútil la preocupación por las gentes de más allá de nuestras fronteras, a gran distancia física y emocional. Incluso critica a los moralistas que pretenden que los agentes se sientan obligados para con toda forma de necesidad en cualquier parte del globo, señalando que dicha preocupación no tiene ningún fin en la medida que carecemos de capacidad de influencia. Concretamente cuestiona a sus lectores: "¿debemos preocuparnos sobre los habitantes de la Luna?» y responde que "esto no es parte de nuestro deber sobre nuestros congéneres». De igual manera, se pregunta por el valor de las condolencias ante un terremoto en la lejana China; ya que tras expresar los más «sinceros» pésames el individuo en cuestión continuará con su vida común sin mayor preocupación dada la lejanía y desconocimiento de estos seres humanos (TSM. III, 3). Este aspecto resulta especialmente interesante en tanto que establece los límites de las esferas en función de la posibilidad de actuación, negando que un hombre pueda experimentar simpatía hacia toda la humanidad (TSM. III, 3.9).

17 Al respecto puede verse Becker (1991). Este tipo de análisis ha tenido también su traducción en el ámbito de la reflexión moral, particularmente desde las perspectivas neoconservadoras. Esta idea se maneja comúnmente, tanto en la sociología como en la politología al considerar los efectos deletéreos del mercado en las instituciones sociales. 
simpatía. En particular, un denominador común dentro de la escolástica smithiana es la no inclusión de la noción de empatía como una dimensión de la simpatía. Para superar esta limitación, Fontaine (1997) propone una distinción: por una parte, existe un vínculo que nos impulsa a contribuir al bienestar de los próximos (simpatía), pero también existe otro por el que adquirimos conocimiento de aquellos con los que frecuentemente interaccionamos (empatía). La distinción entre simpatía y empatía tiene un fundamento en el origen de ambos términos. El término simpatía se refiere a aquellos casos en los que la preocupación por otros afecta directamente a su propio bienestar individual («sentir con»). Empatía, en cambio, ha venido siendo considerada sinónimo de simpatía en el sentido de interdependencia de las funciones de utilidad, aunque está más vinculada al proceso imaginativo de situarse en el lugar de una persona concreta («ponerse en lugar de»). La empatía supone que los sujetos pueden situarse o considerar las circunstancias de otros y predecir sus reacciones (identificación empática, TSM, III, 1) lo que les conduce a considerar su bienestar. En suma, por una parte existe una capacidad de identificación cognitiva o empatía y, por otra, una capacidad de identificación de estado o simpatía (TSM, VII, 3) ${ }^{18}$.

De lo anterior podríamos concluir la existencia de al menos dos dimensiones bajo el término de simpatía: comunicación y predicción e interdependencia de las funciones de utilidad. Esta consideración encuentra también fundamentos en los estudios de psicología de masas en lo que Fodor (1987) consideraba la teoría del sentido común o del deseo-creencia (belief-desire theory). Bajo esta visión, los seres humanos, incluso de diferentes culturas, disponen de una capacidad empática cuasi-innata de predicción y simulación de comportamientos que constituye el principal soporte de la sociabilidad (Gordon, 1998) ${ }^{19}$. Junto a ello, un importante conjunto de estudios en psicología de la economía ha puesto de manifiesto como los individuos están predispuestos hacia la cooperación y simpatía incluso en encuentros con desconocidos y sin posibilidad de efecto reputación (Kahneman, 1994). Más recientemente los modelos conductuales han buscado las bases fisiológicas de estos comportamientos en sustratos neurales capaces de generar un doble sistema de intercambio entre individuos (egoístico-empático) (Cory, 2006).

Por tanto, a la luz de lo señalado serían difícilmente sostenibles los planteamientos que postulan la delimitación precisa de esferas de simpatía, ni la

18 Entre los autores que no realizan la mencionada distinción podemos destacar a Nieli (1986), Heilbroner (1982) o Pack, (1991).

19 Una referencia fundamental en estos estudios son los trabajos de Fodor (1987), así como, Martín y Stone (1995). La propuesta general de esta teoría defiende la existencia de una capacidad natural de inferencia semejante a la gramática universal chomskiana Esta orientación de pensamiento puede considerarse predominante tanto en el ámbito de la filosofía como en el de las ciencias cognitivas. En la ciencia económica merece la pena destacar las investigaciones desarrolladas por la Behavioral Economic Theory y sus extensiones en la Teoría de Juegos (Camerer, 1997; Sally, 2000). 
distinción entre mercado y no-mercado. Es decir, sociedad y mercado forman un continuum donde partiendo de una capacidad de empatía, algunos individuos desarrollan funciones de utilidad interdependientes con los más próximos, sean estos individuos o grupos. Estos vínculos de socialidad son una base elemental de las relaciones de mercado. Si bien ambos modos de identificación, simpatía y empatía, parecen estar aparentemente ausentes de la RN, lo cierto es que Smith era perfectamente consciente del peligro que suponía el establecimiento de relaciones en las que cupiese la posibilidad de defección, en particular los acuerdos esporádicos, por lo que en el fondo subyace siempre un problema de identificación empática.

El problema del desconocimiento o de la carencia de empatía se diluye siempre que exista el sometimiento a una norma, pero sólo cuando la regulación y los contratos sean completos. Sin embargo, esta situación es del todo imposible, de forma que, aunque el intercambio tiene como fundamento el interés particular, la empatía desempeña también un papel crucial en la capacidad de persuasión. De este modo, si el panadero desea hacer una oferta interesante al carnicero necesita ponerse en su situación y considerar lo que haría bajo sus circunstancias. Así, el modo en el que Smith describe la llamada hacia el interés particular de la otra parte en la relación de intercambio sugiere que está considerando un cierto conocimiento sobre las preferencias de aquélla, y parece presuponer la pertenencia a un mismo grupo genérico o la existencia de una identidad básica que permita precoordinar los intereses.

Por consiguiente, la simpatía y el orden moral derivado representan un papel esencial en el ámbito de la Economía. Incluso dentro de la RN, donde se afirma que el interés particular constituye el primer principio, éste no es suficiente para que pueda alcanzarse un acuerdo entre las partes, por lo que siempre existe un trasfondo de simpatía o socialidad elemental que constituye un sistema de precoordinación de las relaciones de mercado.

Estos presupuestos están claramente presentes en la TSM.

"Una sociedad de personas puede subsistir, como la de comerciantes, en razón de la utilidad mutua, sin ningún amor o afecto mutuo (...) por un intercambio mercenario. Pero la sociedad no puede subsistir entre quienes están constantemente prestos a herir a los otros» (TSM, II, 2).

Aunque no es objeto de este trabajo, cabe señalar que esta relación podría ser objeto de una lectura en sentido inverso. Smith también reconoce la incidencia de las relaciones comerciales sobre las relaciones de simpatía, al establecer repetidamente en la $\mathrm{RN}$ una analogía entre la amistad y el comercio cuando afirma que «el comercio debe ser, tanto entre las naciones como entre los individuos, un lazo de unión y amistad» (RN, IV, 3).

La insistencia en la RN afirmando que la benevolencia no constituye la base sobre la que se asienta el intercambio mutuamente benéfico, no es equivalente a expulsar a la simpatía de la esfera de la actividad económica. Tal y 
como concluía Macfie (1976, p. 76), los individuos en sus acuerdos económicos actúan bajo los dictados de la simpatía y del espectador imparcial, y sólo un escaso porcentaje se limita a obedecer los dictados de la justicia. Mediante la simpatía, la situación de los otros repercute en el proceso de toma de decisiones por parte del agente. La simpatía contribuye además a la búsqueda de acuerdos que sean mutuamente benéficos y potencia la división del trabajo. Es decir, los intercambios se asientan en la capacidad comunicativa del hombre (Frantz, 2000, p. 156), capacidad esta que es tanto la base del sistema social y del como del progreso económico ${ }^{20}$.

Así, con arreglo a este planteamiento se pueden distinguir dos tipos de vínculos. Por un lado, están aquellos en los que predomina la simpatía y la relación está regida por la influencia del bienestar ajeno en el propio. Por otro, están las relaciones en las que existe un predominio del interés particular, pero en las que subsiste un vestigio de simpatía-empatía entre los sujetos como base de la comunicación. El mercado, consecuentemente, es también, un ámbito de encuentro social en el cual la simpatía del espectador imparcial es un factor operativo de comprensión de la actividad, la formación de los precios y la distribución (Fleischaker, 2004).

Esta interpretación deja cauces abiertos para incorporar aspectos de la socialidad que tradicionalmente no han sido abordados al analizar los comportamientos económicos. Así, de la noción de simpatía se pueden derivar importantes implicaciones adicionales, como por ejemplo la aparición de sentimientos de pertenencia, identidad o comunidad (Sugden, 2002; Anderson, 2001) ${ }^{21}$, sentimientos que difícilmente podrían ser incorporados a los modelos formales al uso en el marco de la Elección Racional basados exclusivamente en formas de utilidad interdependiente ${ }^{22}$. En realidad, la vida social humana se articula a través del intercambio de sentimientos de correspondencia que pueden acabar por configurar formas de razonamiento en las que los sujetos que forman parte de una comunidad de fellow-fellings razonan colectivamente como si se tratase de un único agente, sujeto plural o pensamiento de equipo (we rationality) (Bacharach, 1999) y extraen utilidad de la propia pertenencia.

Esta última postura hermenéutica resuelve algunos de los aspectos objetables de las dos perspectivas previas: la moral fundamentada en la simpatía como corrector externo y la simpatía como definidor de áreas de no mercado (véase tabla 1). En primer lugar porque la simpatía y la moral basada en la

20 Este argumento también ha sido desarrollado por Buchanan (1996, pp. 124-146).

${ }^{21}$ Esta idea ha sido fuertemente reivindicado por A.K. Sen y resulta esencial en el análisis de la incidencia de la cultura en la Economía. Al respecto puede verse Sen (1985).

22 Esta perspectiva de análisis es muy semejante a la adoptada por Benedetto Gui en su estudio de los bienes relacionales. Para este autor las relaciones sociales tienen un valor subjetivo no instrumental para sus participantes (Gui, 2000). Este tema también es abordado en Hollis (1998). 
misma están también imbricadas en las relaciones de mercado o de interés particular y desempeñan un importante papel en el proceso de toma de decisiones. En segundo lugar, porque se distingue una doble dimensión dentro de la simpatía smithiana, una dimensión emocional o afectiva, por la que funciones de utilidad se hacen interdependientes y una dimensión cognitiva, por la que se accede al modo de pensar del otro. Por último, permite justificar una serie de propiedades emergentes asociadas a la simpatía y que podríamos calificar como de identidad colectiva.

Tabla 1. Propuestas de comprensión de la interrelación entre moral y economía

\begin{tabular}{|c|l|l|c|}
\hline $\begin{array}{c}\text { Propuesta } \\
\text { hermenéutica }\end{array}$ & \multicolumn{1}{c|}{ Principal rasgo } & \multicolumn{1}{c|}{$\begin{array}{c}\text { Disonancia } \\
\text { interpretativa }\end{array}$} & Autores clave \\
\hline $\begin{array}{c}\text { La moral como } \\
\text { de juego. }\end{array}$ & $\begin{array}{l}\text { La simpatía y la } \\
\text { moral construyen las } \\
\text { reglas del juego de } \\
\text { los intereses } \\
\text { particulares. }\end{array}$ & $\begin{array}{l}\text { La simpatía y la } \\
\text { moral constituyen } \\
\text { un elemento externo } \\
\text { a la misma } \\
\text { economía. }\end{array}$ & $\begin{array}{c}\text { Macfie } \\
\text { Cambell } \\
\text { Wilson } \\
\text { Anspach }\end{array}$ \\
\hline $\begin{array}{c}\text { La simpatía y la } \\
\text { moral como sistema } \\
\text { delimitativo de } \\
\text { ámbitos. }\end{array}$ & $\begin{array}{l}\text { La simpatía define } \\
\text { esferas o entornos al } \\
\text { margen del interés } \\
\text { particular donde } \\
\text { predominan otro } \\
\text { tipo de relaciones. }\end{array}$ & $\begin{array}{l}\text { La definición de } \\
\text { estos entornos se } \\
\text { difumina. La } \\
\text { simpatía en diversos } \\
\text { grados existe tras } \\
\text { cualquier relación. }\end{array}$ & $\begin{array}{c}\text { Nieli } \\
\text { Bollander } \\
\text { Becker }\end{array}$ \\
\hline $\begin{array}{c}\text { Simpatía-empatía y } \\
\text { la noción de } \\
\text { identidad. }\end{array}$ & $\begin{array}{l}\text { La simpatía y la } \\
\text { empatía subyacen a } \\
\text { cualquier modo de } \\
\text { interrelación. La } \\
\text { moral es parte } \\
\text { consustancial de la } \\
\text { economía. Se hace } \\
\text { esencial la noción } \\
\text { de identidad. }\end{array}$ & $\begin{array}{l}\text { La inconsistencia se } \\
\text { presenta ahora en la } \\
\text { propuesta de una } \\
\text { moral «heroica» }\end{array}$ & $\begin{array}{c}\text { Sugden } \\
\text { Evensky } \\
\text { Hollis } \\
\text { Young }\end{array}$ \\
Fleischacker \\
\end{tabular}

Fuente: Elaboración propia.

\section{Conclusiones}

El denominado «problema de Smith» es fruto de lecturas positivistas que no consideran el contexto general, ni las interrelaciones existentes entre las distintas partes del programa de investigación smithiano. En realidad, puede comprobarse que existen muy importantes analogías entre la configuración social propuesta en la TSM y la armonía del mercado de la RN, hasta el punto de ser parte de un mismo paradigma. 
La revisión llevada a cabo en el presente trabajo nos permite sistematizar las diversas perspectivas hermeneúticas que han tratado de integrar la obra smithiana. Dichas perspectivas se pueden clasificar atendiendo a un orden lógico establecido en función del papel desempeñado por la simpatía, orden que además guarda relación directa con las diversas interpretaciones que ha tenido el papel de la Ética en la Economía. En concreto, la alternativa interpretativa del conjunto de la obra smithiana basada en la simpatía como base del orden social y propuesta en el presente trabajo como más consistente permite evidenciar cómo la moral configura un sistema de coordinación previo sobre el que se sustenta la coordinación generada por las interacciones del mercado.

La exitosa metáfora de la «mano invisible» de la RN está presente también, aunque implícitamente, en la TSM. Si en la TSM la simpatía y el espectador imparcial controlan las pasiones y los juicios morales de manera que los hombres se orienten hacia la construcción de la sociedad, en la RN el interés particular logra que los individuos sirvan simultáneamente a la sociedad. Es más, a nuestro juicio, la mano invisible representa un papel más directo en la TSM que en la RN, ya que el imaginario que sustenta la sociedad liberal supone no sólo la coordinación de los intereses individuales sino también la de los individuos como homines ethici o miembros de un constructo social. Dicho de otro modo, la construcción de la sociedad liberal clásica se asienta sobre la adhesión a una determinada ética social mínima no impuesta, pero sí compartida.

Las instituciones surgen de las interacciones reiteradas entre individuos asociadas a un sentimiento de simpatía y, por tanto, con un carácter subracional. Consecuentemente, la simpatía desempeña un papel crucial en la coordinación social que supone el mercado. Por lo tanto, la simpatía es una precondición de las interacciones humanas, un compromiso emocional como condición necesaria para la cooperación, que constituye, a su vez, la base para la emergencia de reglas. Por lo tanto De entre las dos aproximaciones posibles al estudio del sistema de mercado, la constructivista o contractualista y la defensora de un orden espontáneo, para la que las instituciones emergen como una consecuencia natural del proceso de mercado, Smith se sitúa claramente en esta última.

Si se invierte la perspectiva de análisis tomando como punto de partida la RN cabe una lectura recíproca: el sistema de mercado es la base necesaria del desarrollo de la moral (Herbener, 1987). Cada participante en el mercado, donde existe un enorme grado de interdependencia, debe ganar la cooperación voluntaria de la otra parte por un proceso de empatía que le lleva al conocimiento de cómo inducir el intercambio. El resultado de esta moderación mutua es un sistema de interacciones, análogo al mecanismo moral de generación de la propia sociedad. En contraste con este escenario, el mercantilismo no permite el desarrollo de la moral, dado que el establecimiento 
de relaciones compulsivas y regladas no da lugar ni al juego de la simpatía-empatía, ni siquiera a la aparición del problema moral (TSM, I, 1, 5). Para Smith, una sociedad de contratos es el fermento apropiado para la moral y, por tanto, si la libre competencia y contratación es la condición de posibilidad del sistema de mercado, la libertad moral lo es de la sociedad liberal.

En definitiva, la simpatía se erige en un concepto articulador que constituye una base sólida para una reflexión ética sobre los hechos económicos desde una racionalidad socioeconómica. Por lo tanto, el problema de Smith puede considerarse como una consecuencia de la identificación errónea entre benevolencia y simpatía. La simpatía es perfectamente compatible con el interés particular y tanto en la TSM como en la RN subsiste el mismo modelo humano. En ultima instancia, tomando como referencia la noción smithiana de simpatía es posible una corrección del paradigma tradicional en Economía y se puede imbricar tanto la ética y los comportamientos prosociales como el interés particular en el proceso de toma de decisiones. En otras palabras, el sistema económico condiciona la moral y, a su vez, la moral es determinante del funcionamiento del sistema económico.

\section{BIBLIOGRAFÍA}

Alvey, J. E. (2003): Adam Smith: Optimist or Pessimist?, Ashgate, Aldershot.

ANDERSON, E. (2001): «Unstrapping the straitjacket of preference», Economics and Philosophy 17, 21-38.

ANSPACH, R. (1972): «The implications of the theory of moral sentiments for A. Smith's economic thought», History of Political Economy 4, 176-206.

BACHARACH, M. (1999): «Interactive team reasoning: a contribution to the theory of cooperation», Research in Economics 53, 117-147.

BECKER, G. (1981): «Altruism in the family and shellfishness in the market place», Economica 48, 1-15.

BECKER, G. (1991): A treatise on the family, Cambridge, Harvard University press.

Brown, V. (1994): A. Smith discourse, canonicity, comerce and conscience, Nueva York, Routledge, 1994.

Buchanan, J. (1996): Ética y progreso económico, Barcelona, Ariel, 1996.

CAMERER, C. F. (1997): «Progress in behavioural game theory», The Journal of Economic Perspectives 11, 4, 167-188.

CAmpBell, W. F. (1967): «A. Smith theory of justice, prudence and beneficence», American Economic Review, 57, 571-577.

CANNAN, E. (ed.) (1896): Lectures on justice, police, revenue and arms, delivered in the university of Glasgow by A. Smith, Oxford, Oxford University Press.

CoRY, G. (2006): «A behavioral model of the dual motive approach to behavioral economics and social exchange», The Journal of Socio-Economics 35, 592-612. 
DANNER, P. L. (1976): «Sympathy and exchangeable value: keys to A. Smith social Philosophy», Review of Social Economy 34, 3, 317-331.

Dimand, M. y Dimand, R. (1991): «Moral sentiments and the marketplace: the consistency of The Theory of Moral sentiments and The Wealth of Nations», en W. BARBER, Perspectives of the economic thought, E. Elgar.

FitzGibBons, A. (1995): Adam Smith's system of liberty, wealth and virtue, Oxford, Oxford University press.

Fleischacker, S. (2004): On Adam Smith's Wealth of Nations: A philosophical companion, Princeton University Press, Princeton.

FoDOR, J. (1987): Psicosemantics: the problem of meaning in philosophy of mind, Cambridge, MIT press, 1987.

FonTAINE, Ph. (1997): «Identification and economic behaviour: simpathy and empathy in historical perspective», Economics and Philosophy, 13, 261-280.

FrAnTZ, R. (2000): «Intuitive elements in A. Smith», Journal of Socioeconomics 29, $1,1-19$.

Fuertes, J. (2000): «La concepción ética de A. Smith», en J. R. LóPeZ y C. CAMPO, Crisis de valores y de normas a finales del siglo XX, Madrid, P. S.

GORDON, R. M. (1998): Sympathy, simulation and the impartial spectator, University of Missouri, St. Louis.

GuI, B. (2000): «Beyond the transactions: on the interpersonal dimension of economic reality», Annals of Public and Cooperative Economics, 71, 139-169.

HAYEK, F. A. (1984): Individualism; true and false (reprint) The essence of Hayek, Stanford, Hoover institute.

HeIlbRONER, R. (1982): «The socialization of individual in A. Smith», History of Political Economy 14, 427-439.

Herbener, J. (1987): «An integration of the Wealth of Nations and The Theory of Moral Sentiments», The Journal of Libertarian Studies, vol. VIII, 2, 276-281.

Hollander, S. (1973): The Economics of A. Smith, Toronto, Toronto University Press.

Hollis, M. (1998): Trust within reason, Cambridge University Press.

IngRaO, B. y RANChetTI, F. (2000): Il mercato nel pensiero economico, Milano, Hoepli, 2000.

KAHNEMAN, D. (1994): «New challenges to the rationality asumption», Journal of Institutional and Theoretical Economics, 150, 18-36.

KhaliL, E. L. (1990): «Beyond self-interest and altruism, a reconstruction of A. Smith's theory of human conduct», Economics and Philosophy, 6, 255-273.

- (2001): «Adam Smith and three theories of altruism», Lovain Economic Review, 67, 4, 421-435.

Kolm, C. (1983): «Altruism and efficiency», Ethics 94, 18-65.

LuX, K. (1990): Adam Smith's mistake; how a moral philosopher invented economics and ended morality, Boston, Shambhala.

Macfie, A. L. (1967): The individual in society, papers on A. Smith, London, George Allen.

Mandeville, B. (1997): La fábula de las abejas, México, Fondo de Cultura Económica.

Martin, D. y Stone, T. (ed.) (1995): Mental simulation: readings in Philosophy and psicology, Oxford, Blackwell. 
Matthews, P. H. (2000): An Austrian (mis)reads A. Smith, Vermont, CERGE.

Morrow, G. R. (1927): «A. Smith, moralist and philosopher», Journal of Political Economy, 35, 321-342.

NiELI, R. (1986): «Spheres of intimacy and the A. Smith problem», Journal of the History of Ideas, 47, 4, 611-624.

ONCKEN, A. (1897): «The consistency of A. Smith», Economic Journal, 7 443-450.

PACK, S. J. (1991): Capitalism as a moral sistem; A. Smith critique of the free market economy, Aldershot, E. Elgar.

PenA, J. A. y SÁnchez, J. M. (2006): «Altruismo, simpatía y comportamientos prosociales en el análisis económico», Principios de Economía Política, n. ${ }^{\circ} 4$, $55-73$.

Peters-Fransen, I. (2001): «The canon in the history of the A. Smith problem», en E. Forget y S. PEART, Reflections on the Classical Canon in Economics, London, Routledge.

RAE, J. (1895): Life of A. Smith, London, Mcmillan.

Raphael, D. (1975): «The Impartial Spectator», en Skinner, A. S. y T. WiLson (edit.) (1975): Essays on Adam Smith, Oxford, Clarendon Press, pp. 83-99.

REeder, J. (1998): «Estudio preliminar», a A. SMith, Ensayos filosóficos, Madrid, Pirámide, pp. 9-39.

Rothbard, M. (2003): Historia del Pensamiento Económico, Madrid, Unión Editorial, vol. I.

SALLY, D. (2000): «A general theory of sympathy, mind reading, and social interaction, with and aplication to the prisoner's dilema», Social science information, 39, 4, 568-634.

SEN, A. K. (1985): «Goals commitment and identity», Journal of Law, Economics and Organization, 1, 2, 343-355.

- (1986): «A Smith's Prudence», en S. HALl y F. STEWARD, Theory and reality in development, London, Mcmillan.

SkInNER, A. (1971): «Introduction», en A. SMith, The Wealth of Nations, London, Penguin.

Skinner, A. S. y WiLson, T. (edit.) (1975): Essays on Adam Smith, Oxford, Clarendon Press.

SMith, A. (1776): Investigación sobre la naturaleza y causas de la riqueza de las naciones, México, Fondo de Cultura Económica, 1992, 7. a edic.

- (1759): La teoría de los sentimientos morales, Madrid, Alianza, 1997.

SugDEN, R. (2002): «Beyond sympathy and empathy: Adam's Smith's concept of fellow-feeling», Economics and Philosophy, 18, 63-87.

TeICHgRAeBer, R. (1986): "Free trade», and moral philosophy: rethinking the sources of A. Smith wealth of nations, Durham, Duke University Press.

VINER, J. (1927): «Adam Smith and the laissez faire», Journal of Political Economy, $35,198-232$.

Walsh, V. (1990) voz: «Philosophy and economics», en J. EATwell y otros (ed.), New Palgrave Dictionary on Economics, vol. 3.

West, E. G. (1976): Introduction to the TMS, Indianapolis, Liberty press.

Wightman, W. P. (1975): «Adam Smith and the history of ideas», en SkInNER, A. S. y Wilson (ed.), Essays on Adam Smith, Oxford University Press. 
WiLson, T. (1975): «Sympathy and self-interest», en Skinner, A. S. y T. WiLson (edit.) (1975), Essays on Adam Smith, Oxford, Clarendon Press, pp. 73-111.

Winch, D. (1978): Adam Smith's politics: an essay in historiografic revision, Cambridge, Cambridge University Press.

Young, J. T. (1997): Economics as a moral science, the political economy of $A$. Smith, Chelteham, E. Elgar Pub. 